\title{
Serum Albumin and Future Risk of Hip, Humeral, and Wrist Fractures in Caucasian Men: New Findings from a Prospective Cohort Study
}

\author{
Setor K. Kunutsor ${ }^{a, b}$ Ari Voutilainen ${ }^{c}$ Michael R. Whitehouse ${ }^{a, b}$ \\ Samuel Seidud, e Jussi Kauhanen ${ }^{c}$ Ashley W. Blom ${ }^{a, b}$ Jari A. Laukkanen ${ }^{c, f, g}$ \\ ${ }^{a}$ National Institute for Health Research Bristol Biomedical Research Centre, University Hospitals Bristol NHS

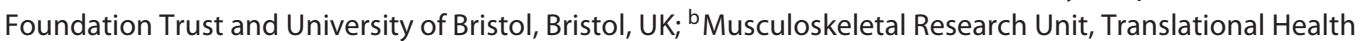 \\ Sciences, Bristol Medical School, University of Bristol, Learning and Research Building (Level 1), Southmead \\ Hospital, Bristol, UK; ' Institute of Public Health and Clinical Nutrition, University of Eastern Finland, Kuopio, Finland;

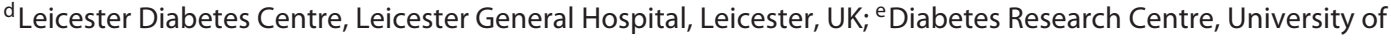 \\ Leicester, Leicester General Hospital, Leicester, UK; ${ }^{f}$ Faculty of Sport and Health Sciences, University of Jyväskylä, \\ Jyväskylä, Finland; ${ }^{9}$ Central Finland Health Care District, Jyväskylä, Finland
}

\section{Significance of the Study}

- Low serum albumin concentration is associated with osteoporosis or low bone mineral density, but its link to fractures has not been extensively investigated. In this study, low serum albumin was associated with an increased risk of long-term fractures in middle-aged Caucasian men, in a linear doseresponse manner. Avoiding low serum albumin levels could help reduce the occurrence of fractures.

\section{Keywords}

Serum albumin · Fracture

\begin{abstract}
Objective: Low serum albumin concentration is associated with poor health outcomes, but its relationship with the risk of fractures has not been reliably quantified. We aimed to assess the prospective association of serum albumin with the risk of fractures in a general population. Subjects and Methods: Baseline serum albumin concentrations were measured in 2,245 men aged 42-61 years in the Kuopio Ischemic Heart Disease study. Hazard ratios (HRs) (95\% confidence intervals) were calculated for incident fractures. $\boldsymbol{R} \boldsymbol{e}$ sults: A total of 121 fractures (hip, humeral, or wrist) were
\end{abstract}

\begin{tabular}{ll}
\hline KARGER & $\begin{array}{l}\text { ( 2019 The Author(s) Karger } \\
\text { Published by S. Karger AG, Basel }\end{array}$ \\
E-Mail karger@karger.com & $\begin{array}{l}\text { This is an Open Access article licensed under the Creative Commons } \\
\text { Attribution-NonCommercial-4.0 International License (CC BY-NC) } \\
\text { (http://www.karger.com/Services/OpenAccessLicense), applicable to } \\
\text { the online version of the article only. Usage and distribution for com- } \\
\text { mercial purposes requires written permission. }\end{array}$
\end{tabular}

recorded during a median follow-up of 25.6 years. The risk of fractures increased linearly below a serum albumin concentration of $\sim 48 \mathrm{~g} / \mathrm{L}$. The age-adjusted HR $(95 \% \mathrm{Cl})$ for fractures per 1 standard deviation lower serum albumin was 1.24 (1.05-1.48). On further adjustment for several conventional and emerging risk factors, the HR was attenuated to 1.21 (1.01-1.45). Comparing the bottom versus top quartile of serum albumin levels, the corresponding adjusted HRs were 2.48 (1.37-4.48) and 2.26 (1.23-4.14). The association of serum albumin with fracture risk did not differ substantially according to age, body mass index, blood pressure, physical activity, alcohol consumption, socioeconomic status, inflammation, prevalent diseases, and smoking. Serum albumin at a threshold of $41.5 \mathrm{~g} / \mathrm{L}$ demonstrated an area under the curve of 0.5850 . Conclusion: In middle-aged Caucasian men,
Setor K. Kunutsor

Musculoskeletal Research Unit, Translational Health Sciences Bristol Medical School, University of Bristol

Learning and Research Building (Level 1)

Southmead Hospital, Bristol BS10 5NB (UK), E-Mail skk31@ cantab.net 
low serum albumin is associated with an increased risk of future fractures. The potential relevance of serum albumin concentrations in fracture prevention and prediction deserves further evaluation.

(c) 2019 The Author(s)

Published by S. Karger AG, Basel

\section{Introduction}

Fractures (particularly osteoporotic fractures) are one of the leading worldwide causes of disability among the aging population and are associated with high health costs [1]. Fractures and their management are associated with considerable pain, limited function, morbidity (e.g., pneumonia, heart failure), reduction in health-related quality of life, as well as mortality $[2,3]$. The prevention of fractures is an issue of public health importance. There is therefore a priority to identify and evaluate risk factors (particularly potentially modifiable ones) that may have predictive or causal relevance, potentially to help tailor preventive and therapeutic strategies. Suggested risk factors for osteoporotic fractures include advancing age, current smoking, high alcohol consumption, glucocorticoid therapy, a family history of fracture, as well as decreased bone mineral density and occurrence of falls [4, 5]. Malnutrition has been identified as a risk factor for poor outcomes following orthopedic surgery [6]. Low serum albumin concentration, a historical marker of malnutrition [7], has been consistently linked with adverse health outcomes such as cardiovascular disease, venous thromboembolism, cancer, as well as increased mortality $[8,9]$. A number of studies have also suggested that low serum albumin concentration is associated with functional decline in the elderly $[10,11]$. Studies have shown that patients with hip fracture are frequently malnourished, indicated by their low serum albumin concentrations $[11,12]$.

Given the overall evidence, we hypothesized that people with low serum albumin concentrations will have an increased risk of fractures and may also have risk factors that are associated with increased fracture risk. Data on the temporal nature of the relationship between serum albumin concentrations and fracture risk are limited, as the majority of previous studies evaluated serum albumin concentrations after fracture occurrence. In the only prospective cohort study, which was published over three decades ago, Huang et al. [13] demonstrated an inverse association between serum albumin and hip fracture risk in a sample of Caucasian women. Drawbacks of this study included (a) the inability to account for some potential confounders such as preexisting disease, renal function, energy intake, trace elements, and inflammation; (b) the lack of a formal assessment of the shape of the relationship between serum albumin and fracture risk; hence, it is uncertain whether there is a dose-response relationship to the association; and (c) a lack of subgroup analysis.

In this context, we aimed to investigate in greater detail than before, the shape, nature, and magnitude of the prospective association between serum albumin and risk of future fractures using a population-based cohort of 2,245 apparently healthy men from eastern Finland. We also assessed the consistency of the association in important clinical subgroups such as age, body mass index (BMI), physical activity, and smoking status. Finally, we determined the threshold and discriminative utility of serum albumin concentration for fractures.

\section{Methods}

Study Design and Participants

The analysis employed the Kuopio Ischemic Heart Disease (KIHD) study, which is a population-based prospective cohort set up to evaluate risk factors for cardiovascular disease and other chronic disease outcomes. The cohort, design, and recruitment methods have been described previously $[14,15]$. Briefly, the KIHD cohort is based on a representative sample of 3,433 randomly selected men aged 42-61 years from the city of Kuopio and surrounding rural communities in eastern Finland, who were invited for screening examinations between March 1984 and December 1989. In this analysis, complete information on serum albumin, relevant confounders, and fracture events was available for 2,245 men.

\section{Assessment of Serum Albumin and Risk Markers}

Baseline assessments involved physical examination, collection of blood samples, and the use of self-administered questionnaires. Besides fasting overnight before collection of blood samples in the morning between 08:00 and 10:00 h, participants were also required to abstain from smoking for at least $12 \mathrm{~h}$ and alcohol consumption for at least 3 days prior to blood collection. Serum samples were stored frozen at $-80^{\circ} \mathrm{C}$ for $0.2-2.5$ years before measurements were made. Serum albumin concentrations were measured using Coulter's bromocresol purple colorimetric assay (Kone Specific, Kone Corporation, Espoo, Finland). Serum high-sensitivity C-reactive protein (hsCRP) measurements were made with an immunometric assay (Immulite High Sensitivity C-Reactive Protein Assay; DPC, Los Angeles, CA, USA). The energy expenditure of physical activity was assessed using the validated KIHD 12-month leisure time physical activity questionnaire [14].

\section{Ascertainment of Incident Fractures}

All incident fractures, representing all hip, humeral, and wrist fracture cases that occurred from study entry to 2014 , were includ- 
Table 1. Baseline participant characteristics overall and by quartiles of serum albumin

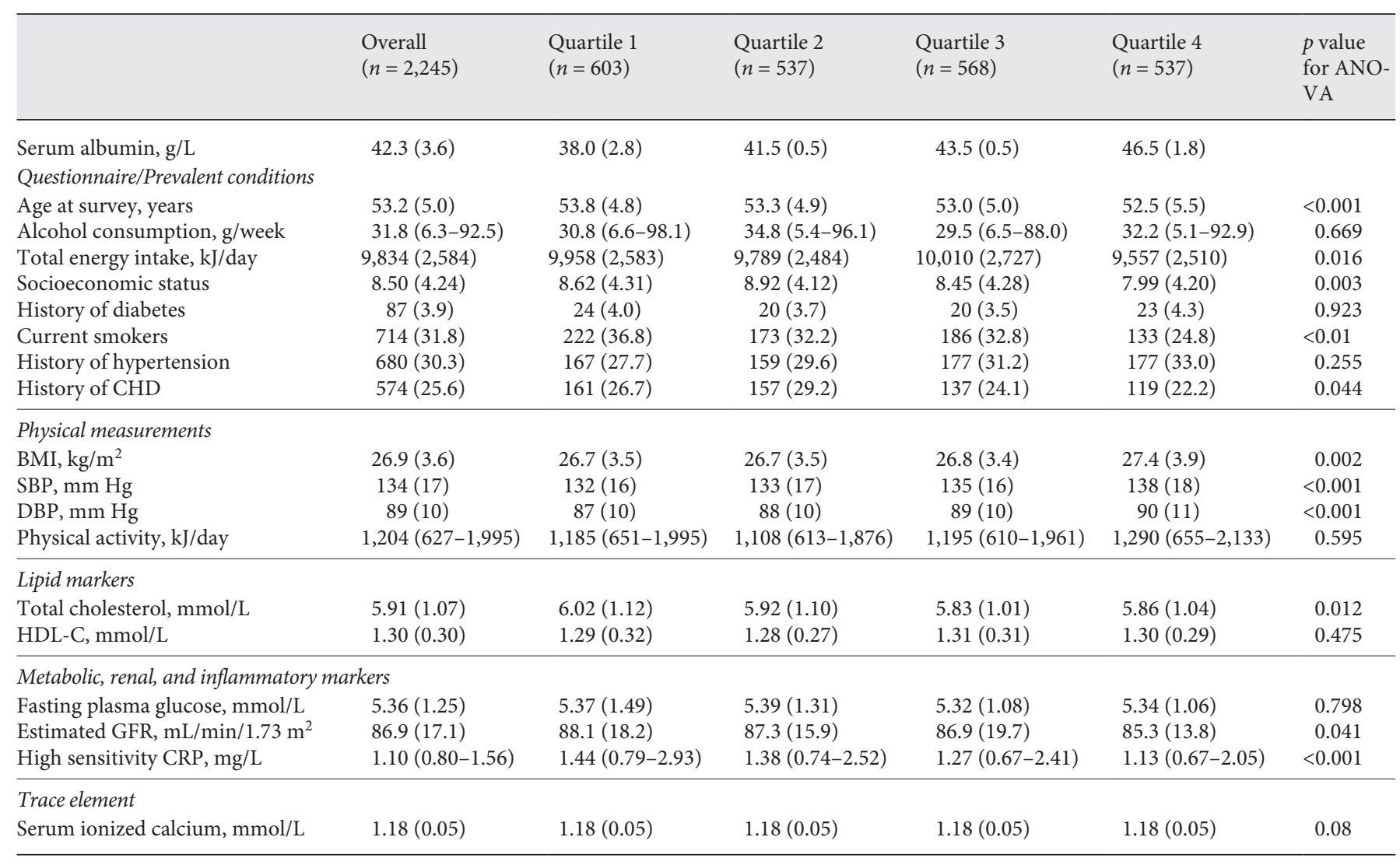

Values are presented as mean (SD), median (IQR) or $n$ (\%). BMI, body mass index; CHD, coronary heart disease; CRP, C-reactive protein; DBP, diastolic blood pressure; GFR, glomerular filtration rate; HDL-C, high-density lipoprotein cholesterol; SD, standard deviation; SBP, systolic blood pressure

ed. The primary outcome measure assessed was any fracture (defined as hip, humeral, or wrist fractures). Data on incident fractures was collected from the National Hospital Discharge Register data by computer linkage using Finnish personal identification codes and a comprehensive review of hospital records, discharge diagnoses, and inpatient physician claims. Outcome events were coded according to the International Classification of Diseases Tenth Revision diagnostic codes for fractures by site.

\section{Statistical Analyses}

Skewed variables were log-transformed to achieve approximately symmetrical distributions. Cox proportional hazard regression models were used to conduct time-to-event analyses after confirming no major departure from the assumptions of proportionality of hazards, using Schoenfeld residuals. To characterize the shape of the association between serum albumin concentrations and fracture risk, we used floating absolute risks as described previously [15]. To further explore the shape of the relationship, we used a restricted cubic spline with knots at the 5th, 35th, 65th and 95th percentiles of serum albumin distribution in a multivariate adjusted model. Serum albumin was modelled continuously, per 1 standard deviation (SD) change (per 1 SD decrement) in serum albumin levels as the relationship between serum albu- min and fracture risk was approximately linear. Hazard ratios (HRs) were also modelled as quartiles defined according to the baseline distribution of serum albumin levels in the sample. The HRs were progressively adjusted for (a) age; (b) established risk factors and other potential confounders (BMI, systolic blood pressure, history of hypertension, prevalent coronary heart disease, smoking status, history of type 2 diabetes, alcohol consumption, physical activity, estimated glomerular filtration rate, as calculated using the Chronic Kidney Disease Epidemiology Collaboration formula, socioeconomic status, energy intake, and serum ionized calcium); and (c) hsCRP. Given the long follow-up period and therefore a high mortality rate which could have hindered our primary outcomes, we also performed additional analyses to estimate the baseline cumulative subhazard of fracture considering all-cause death as a competing outcome to fracture, using the competing risks extension of the Cox proportional hazards models, as proposed by Fine and Gray [16]. We used the receiver operating characteristic curve to determine the best cutoff point of serum albumin which could be used to predict fractures, with the predictive accuracy expressed as area under the curve. All statistical analyses were conducted using Stata version 15 (Stata Corp., College Station, TX, USA). 


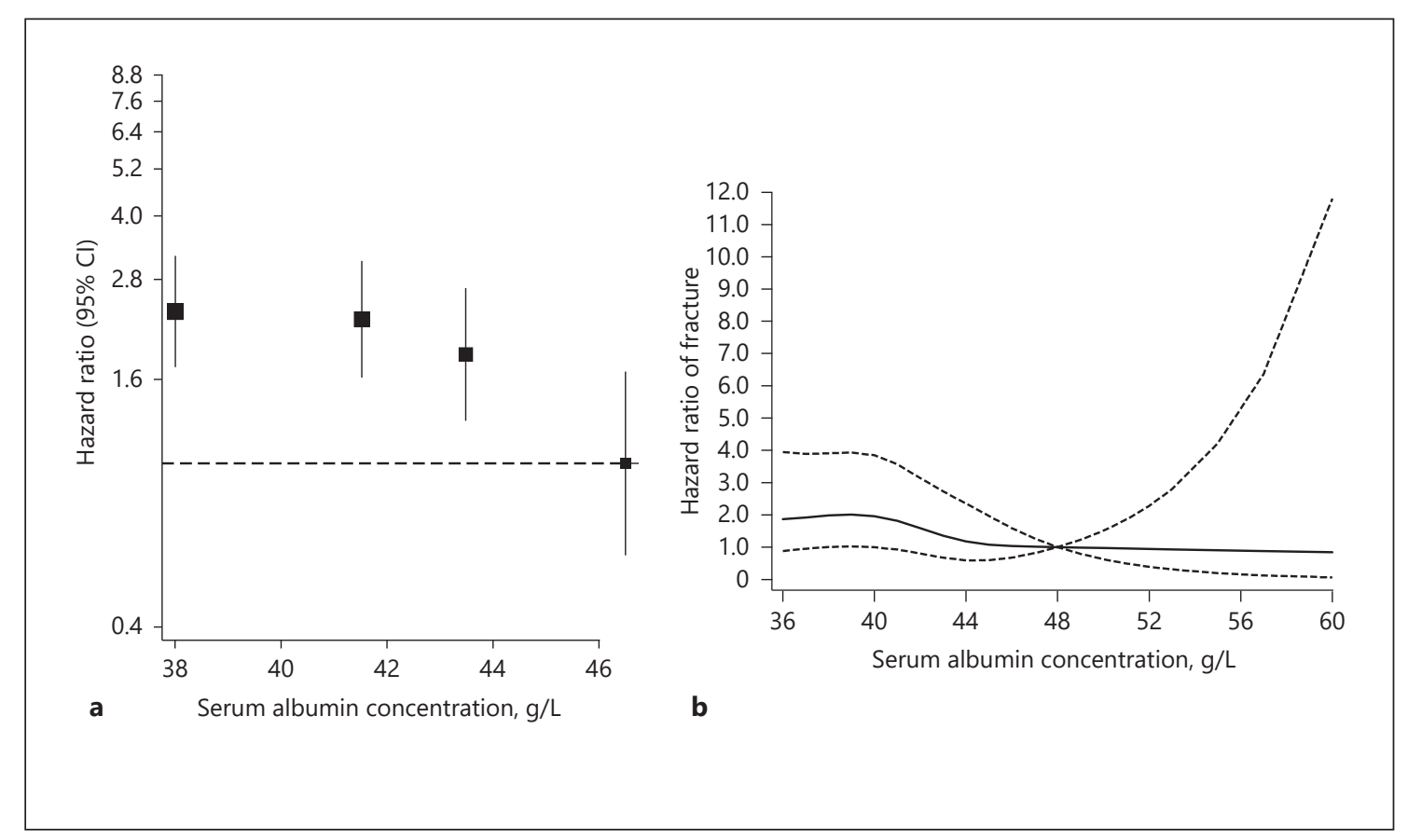

Fig. 1. Shapes of the association between serum albumin levels and incident fractures. a Shape plotted using floating absolute risks; top quartile of serum albumin levels was used as reference. b Restricted cubic spline model of the hazard ratios of composite fracture with serum albumin; restricted cubic spline functions were analyzed with knots located at the 5th, 35th, 65th and 95th percentiles of serum albumin distribution $(37 ; 41 ; 44$; and $47.15 \mathrm{~g} / \mathrm{L})$ with

\section{Results}

\section{Baseline Characteristics}

Baseline characteristics of overall study participants and by quartiles of serum albumin are summarized in Table 1 . The mean (SD) age and BMI of study participants was $53(5)$ years and $26.9(3.6) \mathrm{kg} / \mathrm{m}^{2}$, respectively. The mean (SD) serum albumin level was 42.3 (3.6) g/L. Participants in the bottom quartile of serum albumin levels were older, more likely to be smokers and have preexisting disease such as coronary heart disease and had higher levels of total cholesterol and hsCRP compared with participants in quartiles 2-4. Men with a fracture at followup were older and had lower serum albumin levels at study entry.

\section{Serum Albumin and Risk of Fractures}

During a median follow-up of 25.6 (interquartile range, 17.6-27.9) years, 121 fracture events (annual rate 2.44/1,000 person-years at risk; 95\% CI 2.05-2.92) were recorded. Serum albumin was approximately linearly as- the reference category set at $48 \mathrm{~g} / \mathrm{L}$; the dashed lines represent the $95 \%$ confidence intervals. All models were adjusted for age, body mass index, systolic blood pressure, history of hypertension, prevalent coronary heart disease, smoking status, history of type 2 diabetes, alcohol consumption, physical activity, estimated glomerular filtration rate, socioeconomic status, energy intake, and serum ionized calcium. sociated with risk of fractures (Fig. 1a). A restricted cubic spline curve shows the risk of fractures increasing linearly below a serum albumin concentration of $\sim 48 \mathrm{~g} / \mathrm{L}$ ( $p$ value for nonlinearity $=0.308$ ) and plateaus at a serum albumin concentration of $\sim 40 \mathrm{~g} / \mathrm{L}$ (Fig. 1b). The HR for fractures per $1 \mathrm{SD}$ decrement in serum albumin was 1.24 (95\% CI 1.05-1.48) on adjustment for age which remained unchanged on further adjustment for several established risk factors and other potential confounders. The association remained consistent on additional adjustment for hsCRP 1.21 (95\% CI 1.01-1.45). When the bottom quartile of serum albumin was compared to the top quartile, the corresponding adjusted HRs were 2.48 (95\% CI 1.37-4.48), 2.34 (95\% CI 1.28-4.28), and 2.26 (95\% CI 1.23-4.14), respectively (Table 2 ). A total of 1,124 all-cause deaths occurred during follow-up. In analyses including all-cause death as a competing risk event, the corresponding adjusted HRs for fractures were 2.20 (95\% CI 0.96-5.05), 1.96 (95\% CI 0.83-4.62), and 1.92 (95\% CI 0.81-4.51), respectively, comparing the bottom quartile to the top quartile of serum albumin levels. 


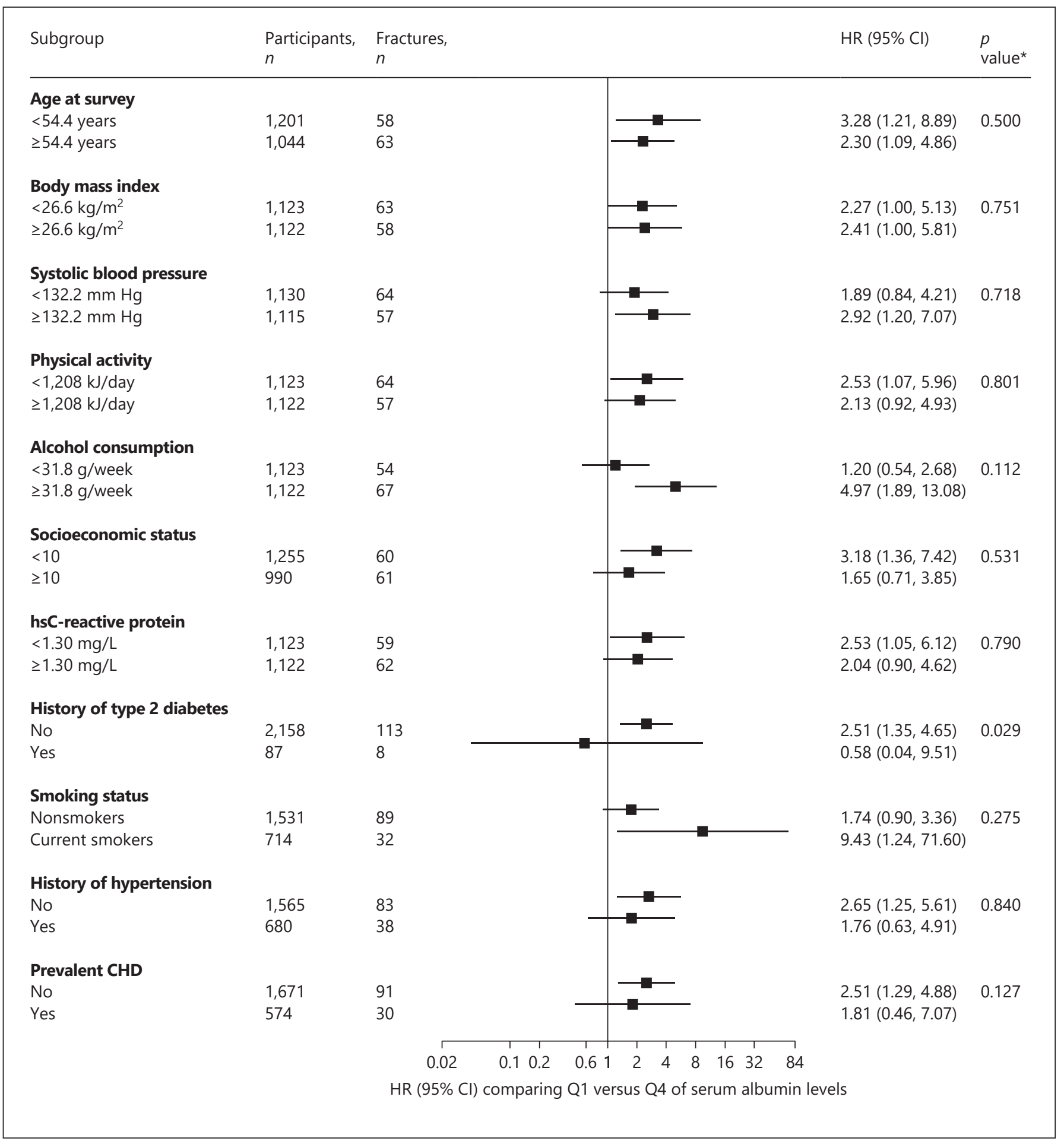

Fig. 2. Hazard ratios for baseline levels of serum albumin and fracture risk by several participant level characteristics. Hazard ratios are adjusted for age, body mass index, systolic blood pressure, history of hypertension, prevalent coronary heart disease, smoking status, history of type 2 diabetes, alcohol consumption, physical activity, estimated glomerular filtration rate, socioeconomic status, energy intake, and serum ionized calcium. CHD, coronary heart disease; $\mathrm{CI}$, confidence interval; $\mathrm{HR}$, hazard ratio; hs, high sensitivity; Q, quartile. * $p$ value for interaction; cutoffs used for age, body mass index, systolic blood pressure, physical activity, alcohol consumption, socioeconomic status, and high-sensitivity C-reactive protein are median values. 
Table 2. Association of serum albumin with fractures

\begin{tabular}{|c|c|c|c|c|c|c|c|}
\hline \multirow[t]{2}{*}{ Serum albumin } & \multirow{2}{*}{$\begin{array}{l}\text { Events/ } \\
\text { total }\end{array}$} & \multicolumn{2}{|l|}{ Model 1} & \multicolumn{2}{|l|}{ Model 2} & \multicolumn{2}{|l|}{ Model 3} \\
\hline & & HR (95\% CI) & $p$ value & HR (95\% CI) & $p$ & HR (95\% CI) & $p$ \\
\hline Per 1 SD decrease & $121 / 2,245$ & $1.24(1.05-1.48)$ & 0.013 & $1.22(1.02-1.47)$ & 0.029 & $1.21(1.01-1.45)$ & 0.041 \\
\hline Q1 (10-40 g/L) & $42 / 603$ & $2.48(1.37-4.48)$ & 0.003 & $2.34(1.28-4.28)$ & 0.006 & $2.26(1.23-4.14)$ & 0.008 \\
\hline Q2 (41-42 g/L) & $36 / 537$ & $2.47(1.35-4.52)$ & 0.038 & $2.24(1.22-4.12)$ & 0.010 & $2.18(1.18-4.01)$ & 0.013 \\
\hline Q3 (43-44 g/L) & $28 / 568$ & $1.94(1.04-3.64)$ & 0.113 & $1.84(0.98-3.47)$ & 0.059 & $1.81(0.96-3.41)$ & 0.066 \\
\hline Q4 (45-60 g/L) & $15 / 537$ & ref & & ref & & ref & \\
\hline
\end{tabular}

CI, confidence interval; HR, hazard ratio; ref, reference; Q, quartile; SD, standard deviation. Model 1: adjusted for age. Model 2: model 1 plus body mass index, systolic blood pressure, history of hypertension (no vs. yes), prevalent coronary heart disease (no vs. yes), smoking status (no vs. current), history of type 2 diabetes (no vs. yes), alcohol consumption, physical activity, estimated glomerular filtration rate, socioeconomic status, dietary energy intake, and serum ionized calcium. Model 3: model 2 plus high-sensitivity C-reactive protein. Except for where indicated, all other confounders were modelled as continuous variables.

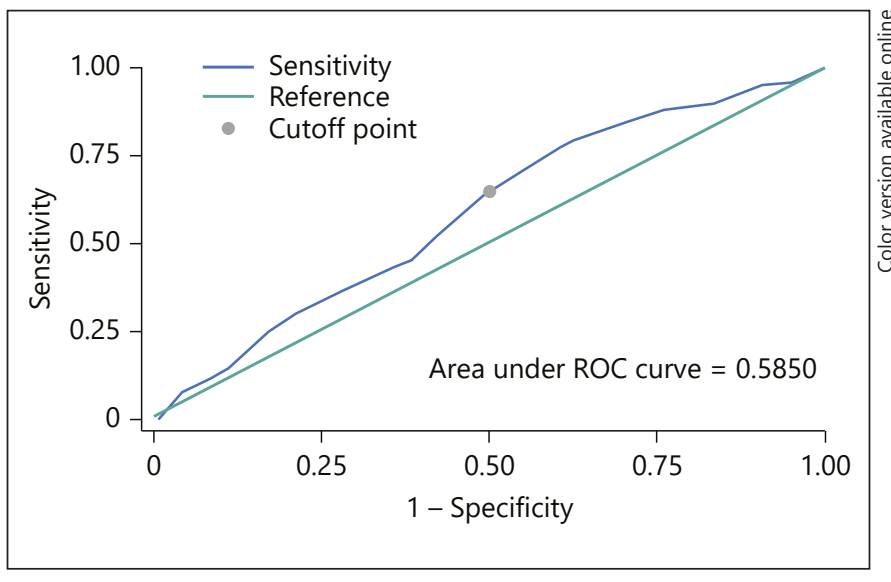

Fig. 3. Receiver operator characteristic curve for serum albumin.

Except for evidence of an interaction by prevalent type 2 diabetes $(p$ for interaction $=0.029$ ), the association between serum albumin and fracture risk was not significantly modified by several clinically relevant characteristics ( $p$ for interaction $\geq 0.10$ for each; Fig. 2 ). The association between low serum albumin and increased fracture risk was stronger in men without a history of type 2 diabetes compared with those with a history of type 2 diabetes.

The receiver operating characteristic curve showed that a serum albumin threshold of $41.5 \mathrm{~g} / \mathrm{L}$ had $64 \%$ sensitivity and $50 \%$ specificity as a predictive marker for fractures. The area under the curve was 0.5850 (Fig. 3).

\section{Discussion}

In this population-based prospective study of middleaged Caucasian men, we found low serum albumin levels to be associated with an increased risk of any fractures as defined by a combined outcome of hip, humeral, and wrist fractures. The association seemed to be largely independent of several conventional and emerging risk factors. The risk of fractures increased in an approximately linear manner at serum albumin concentrations which are lower but within normal reference ranges. The association between serum albumin and fracture risk was modest when all-cause death was adjusted for as a competing risk event. However, this is not surprising, as serum albumin is independently associated with risk of allcause death in our study cohort. The association between serum albumin concentration and fracture risk remained generally consistent across several clinically relevant subgroups, except for evidence of effect modification by a history of type 2 diabetes. Finally, our results showed that a serum albumin threshold of $41.5 \mathrm{~g} / \mathrm{L}$ could be used to predict fractures; however, the discriminative ability is limited.

Various important factors that play a role in bone health and influence fracture risk include aging, heritability, sex, physical activity, hormonal factors, and nutrition [17]. Occurrence of falls and reduced bone mass are also major risk factors for osteoporotic fractures $[4,5]$. The effect of nutrition on bone health and fractures has mostly focused on specific dietary factors such as calcium and vitamin $\mathrm{D}$, and more recently magnesium [15]. The biological basis for the strong link between nutritional status 
and fracture risk is quite clear. Poor nutritional status and deficiencies in nutrients required for bone growth adversely affect the balance between bone formation and resorption, which influences bone fragility and results in osteoporosis [18]. Poor nutritional status also contributes to general bodily weakness, which increases the propensity for falls and subsequent fractures. Though serum albumin is historically considered to be a marker of nutrition [7], the biological pathways linking low serum albumin with an increased risk of future fractures is not clear. However, albumin is present throughout the bone matrix and evidence suggests that circulating albumin may be directly involved in fracture development. The underpinning mechanisms postulated include: (a) hypoalbuminemia may directly activate osteoclasts and inhibit osteogenesis via its relationship with nuclear factor- $\kappa \mathrm{B}[19]$; (b) alteration in the metabolism of parathyroid hormone and vitamin D binding protein [20]; (c) decreased Gla protein resulting in decreased osteoblastic and increased osteoclast activities [21]; and (d) albumin has an anabolic effect on bone metabolism via its stimulatory effect on bone calcium and DNA content [22], hence low serum levels may be detrimental to bone. Low serum albumin level is also strongly linked to functional decline or outcomes $[10,11]$; it is therefore plausible that low serum albumin levels cause an increase in fracture risk via the close relationship with functional decline and occurrence of falls. Furthermore, low serum albumin may just be an indicator of an underlying poor general health and nutritional status $[7,11]$, which increase the risk of fractures [13].

The observed relationship between serum albumin and fracture risk may suggest a causal relationship; however, this remains to be investigated in future studies. The current findings, however, do have implications for clinical practice - avoiding low serum albumin concentrations may be a promising strategy for the prevention of fractures in the general population. This therapeutic potential has been demonstrated in several previous clinical and experimental studies. Serum albumin has been shown to enhance the proliferation of stem cells on bone allograft as well as bone healing in a nonunion in human as well as animal models $[23,24]$. In vitro and in vivo experiments in rat models have shown that serum albumin coating of demineralized bone matrix resulted in faster and stronger new bone formation [25]. The overall evidence suggests that avoiding low serum albumin levels could help reduce the occurrence of fractures. Increasing serum albumin concentrations may protect against the future risk of fractures; however, well-designed supple- mentation trials are needed to investigate these potential therapeutic implications. Furthermore, trials aimed at resolution of the underlying causes of low albumin should also be considered.

Our analyses employed a large-scale populationbased prospective cohort study which comprised men recruited from the general population; there was complete follow-up for all participants; there was a long follow-up period of over 20 years for the ascertainment of outcome data; our analyses were comprehensive, which included adjustment for key confounders, assessment of the dose-response relationship, and evaluation of the association in clinically relevant subgroups; and assay measurements for serum albumin measurements which employed the bromocresol purple assay, which agrees with the gold standard of immunonephelometry [14]. There are important caveats about the present study that are worthy of mention. First, though participants abstained from smoking for at least $12 \mathrm{~h}$ and alcohol consumption for at least 3 days prior to blood collection, this was not sufficient to eliminate the influence of longterm smoking and alcohol consumption on albumin levels. However, evidence suggests that alcohol consumption exhibits acute effects by inhibiting albumin synthesis [26]. Similarly, acute cigarette smoke seems to have a suppressive effect on inflammatory markers [27]. Therefore, by abstaining from smoking for at least $12 \mathrm{~h}$ and alcohol consumption for at least 3 days prior to blood collection, any acute effects of smoking and alcohol consumption on albumin levels would have been minimized, if not eliminated. In addition, our cohort employed approximately healthy participants. Finally, our analysis adjusted for both smoking and alcohol consumption, thereby minimizing any biases by these factors. Second, the relatively low event rate did not provide adequate power which was evidenced by the wide confidence intervals and this also precluded sensitivity analyses, which would require exclusions. Given this, we were also unable to adequately investigate the associations in specific fracture sites. Third, we only had outcome data on fractures related to the hip, humerus, and wrist and therefore could not assess the associations for a broad range of fractures related to osteoporosis. Fourth, the present findings cannot be generalized to women and other age groups than those included herein; however, results from a previous study suggest the current findings may be applicable to women [13]. Fifth, despite careful adjustment for a comprehensive panel of confounders, other potential confounders could have been considered such as prevalent conditions (e.g., thy- 
roid disease, Crohn's disease, celiac disease, myelomas, renal disease, liver disease), previous fracture, use of medications, and other factors associated with bone health such as vitamin $\mathrm{D}$ and parathyroid hormone levels. In addition, there was a potential for residual confounding by adjusted factors such as dietary factors, smoking, and alcohol consumption, due to the lack of granular assessment on their frequency, recentness, and duration of use. Sixth, we had no data on fractures related to falls or fall-related hospitalizations. Finally, we were unable to correct for regression dilution because of absence of repeat measurements of serum albumin, which could have underestimated the associations. The findings of the present study should be interpreted with caution considering these limitations. Further studies are indeed needed to replicate this association.

In summary, the association between low serum albumin and increased fracture risk in middle-aged Caucasian men is consistent with a linear dose-response relationship at serum albumin concentrations which are lower but within normal reference range. The potential relevance of serum albumin concentrations in fracture prevention and prediction deserves further evaluation.

\section{Acknowledgements}

We thank the staff of the Kuopio Research Institute of Exercise Medicine and the Research Institute of Public Health and University of Eastern Finland, Kuopio, Finland, for collecting data.

This work was supported by the Finnish Foundation for Cardiovascular Research, Helsinki, Finland. Drs. Kunutsor, Whitehouse, and Blom acknowledge support from the NIHR Biomedical Research Centre at University Hospitals Bristol NHS Foundation Trust and the University of Bristol. The views expressed in this publication are those of the authors and not necessarily those of the NHS, the National Institute for Health Research or the Department of Health and Social Care. These sources had no role in design and conduct of the study, collection, management, analysis, and interpretation of the data, and preparation.

\section{Statement of Ethics}

The Ethics Review Board of the University of Eastern Finland approved the study protocol. Written informed consent was obtained from the participants and all study procedures were conducted according to the Declaration of Helsinki.

\section{Disclosure Statement}

The authors declare no conflicts of interest.

\section{References}

1 Rachner TD, Khosla S, Hofbauer LC. Osteoporosis: now and the future. Lancet. $2011 \mathrm{Apr}$; 377(9773):1276-87.

2 Johnell O, Kanis JA. An estimate of the worldwide prevalence and disability associated with osteoporotic fractures. Osteoporos Int. 2006 Dec;17(12):1726-33.

3 Richmond J, Aharonoff GB, Zuckerman JD, Koval KJ. Mortality risk after hip fracture. J Orthop Trauma. 2003 Jan;17(1):53-6.

4 Wainwright SA, Marshall LM, Ensrud KE, Cauley JA, Black DM, Hillier TA, et al.; Study of Osteoporotic Fractures Research Group. Hip fracture in women without osteoporosis. J Clin Endocrinol Metab. 2005 May;90(5):2787-93.

5 Kanis JA. Diagnosis of osteoporosis and assessment of fracture risk. Lancet. 2002 Jun; 359(9321):1929-36.

6 Bohl DD, Shen MR, Kayupov E, Della Valle CJ. Hypoalbuminemia Independently Predicts Surgical Site Infection, Pneumonia, Length of Stay, and Readmission After Total Joint Arthroplasty. J Arthroplasty. 2016 Jan; 31(1):15-21.

7 Russell M, McAdams M, Matarese L, et al. Laboratory monitoring of nutritional status. In: Matarese LE, Gottschlich MM, editors. Contemporary nutrition support practice: a clinical guide. Philadelphia: WB Sanders; 1998. p. 47-63.

8 Kunutsor SK, Seidu S, Katechia DT, Laukkanen JA. Inverse association between serum albumin and future risk of venous thromboembolism: interrelationship with high sensitivity C-reactive protein. Ann Med. 2018 May;50(3):240-8.

9 Phillips A, Shaper AG, Whincup PH. Association between serum albumin and mortality from cardiovascular disease, cancer, and other causes. Lancet. 1989 Dec;2(8677):1434-6.

10 Covinsky KE, Covinsky MH, Palmer RM, Sehgal AR. Serum albumin concentration and clinical assessments of nutritional status in hospitalized older people: different sides of different coins? J Am Geriatr Soc. 2002 Apr; 50(4):631-7.

11 Zuliani G, Romagnoni F, Volpato S, Soattin L, Leoci V, Bollini MC, et al. Nutritional parameters, body composition, and progression of disability in older disabled residents living in nursing homes. J Gerontol A Biol Sci Med Sci. 2001 Apr;56(4):M212-6.

12 Fisher A, Srikusalanukul W, Fisher L, Smith PN. Lower serum P1NP/ $\beta$ CTX ratio and hypoalbuminemia are independently associated with osteoporotic nonvertebral fractures in older adults. Clin Interv Aging. 2017 Jul;12: 1131-40.

13 Huang Z, Himes JH, McGovern PG. Nutrition and subsequent hip fracture risk among a national cohort of white women. Am J Epidemiol. 1996 Jul;144(2):124-34.

14 Kunutsor SK, Khan H, Laukkanen JA. Serum albumin concentration and incident type 2 diabetes risk: new findings from a populationbased cohort study. Diabetologia. 2015 May; 58(5):961-7.

15 Kunutsor SK, Whitehouse MR, Blom AW, Laukkanen JA. Low serum magnesium levels are associated with increased risk of fractures: a long-term prospective cohort study. Eur J Epidemiol. 2017 Jul;32(7):593-603.

16 Fine JP, Gray RJ. A Proportional Hazards Model for the Subdistribution of a Competing Risk. J Am Stat Assoc. 1999;94(446):496-509.

17 Martini LA. Magnesium supplementation and bone turnover. Nutr Rev. 1999 Jul;57(7): 227-9.

18 Parfitt AM. Dietary risk factors for age-related bone loss and fractures. Lancet. 1983 Nov: 2(8360):1181-5.

19 Abu-Amer Y. NF- $\mathrm{kB}$ signaling and bone resorption. Osteoporos Int. 2013 Sep;24(9): 2377-86. 
20 Morii $\mathrm{H}$, Shioi A, Inaba M, Goto H, Kawagishi T, Nakatsuka K, et al. Significance of albumin in the pathogenesis of osteoporosis: bone changes in genetically analbuminemic rats and rats fed a low albumin diet. Osteoporos Int. 1997;7(S3 Suppl 3):S30-5.

21 Diamond TH, Stiel D, Lunzer M, McDowall D, Eckstein RP, Posen S. Hepatic osteodystrophy. Static and dynamic bone histomorphometry and serum bone Gla-protein in 80 patients with chronic liver disease. Gastroenterology. 1989 Jan;96(1):213-21.
22 Yamaguchi M, Igarashi A, Misawa H, Tsurusaki Y. Enhancement of albumin expression in bone tissues with healing rat fractures. J Cell Biochem. 2003 May;89(2):356-63.

23 Skaliczki G, Schandl K, Weszl M, Major T, Kovács M, Skaliczki J, et al. Serum albumin enhances bone healing in a nonunion femoral defect model in rats: a computer tomography micromorphometry study. Int Orthop. 2013 Apr;37(4):741-5.

24 Pecina M, Haspl M, Jelic M, Vukicevic S. Repair of a resistant tibial non-union with a recombinant bone morphogenetic protein-7 (rh-BMP-7). Int Orthop. 2003;27(5):320-1.
25 Horváthy DB, Vácz G, Szabó T, Szigyártó IC, Toró I, Vámos B, et al. Serum albumin coating of demineralized bone matrix results in stronger new bone formation. J Biomed Mater Res B Appl Biomater. 2016 Jan;104(1):126-32.

26 Rothschild MA, Oratz M, Schreiber SS. Effects of nutrition and alcohol on albumin synthesis. Alcohol Clin Exp Res. 1983;7(1):2830.

27 van der Vaart H, Postma DS, Timens W, ten Hacken NH. Acute effects of cigarette smoke on inflammation and oxidative stress: a review. Thorax. 2004 Aug;59(8):713-21. 